\title{
Piecing together anxiety
}

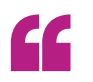

\section{these studies}

reveal that

various

subregions

of the BNST

influence the

manifestation

of anxiety

through the

actions of

opposing local

neural circuits

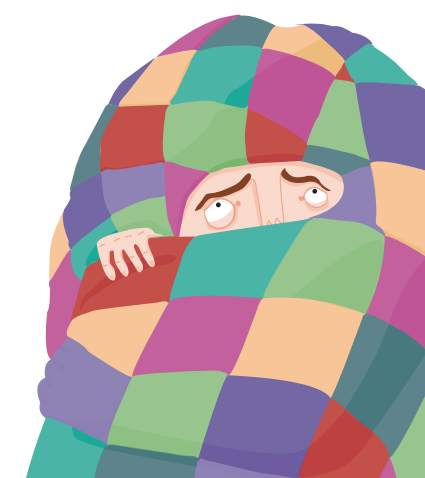

Anxiety comprises various features encompassing behavioural changes, such as increased risk avoidance behaviour, and physiological alterations, such as a rise in the respiratory rate. How these features are represented and, indeed, coordinately regulated at the level of neural circuits remains unclear. Now, two studies reveal the central role that subregions of the bed nucleus of the stria terminalis (BNST) have in the regulation of the anxious state.

The BNST is part of the extended amygdala, and lesions in this nucleus have been linked to decreases in fear- and anxiety-like behaviour in rats. Kim et al. focused their study on two subregions of the dorsal BNST (dBNST) - the oval nucleus of the BNST (ovBNST) and the anterodorsal BNST (adBNST).

Optogenetic inhibition of the ovBNST increased the time that mice spent exploring open spaces - a sign of reduced risk avoidance behaviour. Conversely, optogenetic stimulation of the ovBNST led to increased anxiety-like behaviour and a rise in the respiratory rate, indicating that activity in this subregion promotes anxiety in mice. The basolateral amygdala (BLA) is also implicated in anxiety, and the authors identified BLA pyramidal neurons that specifically projected to the adBNST. Optogenetic stimulation of these BLA-adBNST pathways promoted open space exploration and reduced the respiratory rate, whereas optogenetic inhibition of these pathways had the opposite effects. Thus, in contrast to ovBNST activity, stimulation of the adBNST seems to promote anxiolytic responses.

The authors also found that several distinct neural pathways emanating from the adBNST specifically controlled this area's anxiolytic effects. Stimulation of adBNSTlateral hypothalamus projections decreased avoidance of open spaces but had no effect on respiration, whereas stimulation of adBNSTparabrachial nucleus projections decreased the respiratory rate but had no effect on behaviour. In addition, stimulation of adBNST projections to the ventral tegmental area (VTA), which is involved in aversion and reward, increased place preference (an anxiolytic response) but did not affect risk avoidance behaviour or the respiratory rate.

In contrast to Kim et al., Jennings and colleagues focused their attention on the ventral BNST (vBNST) and its connectivity with the VTA. These authors found that optogenetic stimulation of glutamaterigc and GABAergic vBNST neurons induced excitatory and inhibitory currents in VTA neurons, respectively.

They next examined how these different populations of VTAprojecting
vBNST neurons responded to aversive stimuli, namely unpredictable footshocks, and their contextual cues. Overall, glutamatergic BNST-VTA neurons showed an increase in activity during exposure to such footshocks or their associated contextual cues alone (a model of learned anxiety). By contrast, activity in GABAergic BNST-VTA neurons diminished during exposure to footshocks or their contextual cues.

Both vBNST neuronal populations preferentially formed functional synaptic connections with nondopaminergic neurons in the VTA. Optogenetic activation of vBNST glutamatergic neuronal projections to VTA non-dopaminergic neurons led to decreases in place preference, reward-seeking and exploration of open spaces, whereas activation of vBNST GABAergic neuronal projections promoted these behaviours. Thus, these results show that two subpopulations of vBNST-VTA projections seem to have opposing roles in the generation of reward-seeking and anxiety-like states.

Together, these studies reveal that various subregions of the BNST influence the manifestation of anxiety through the actions of opposing local neural circuits and show that the various features of the anxious state are regulated through seemingly distinct pathways emanating from these circuits.

\section{Darran Yates}

ORIGINAL RESEARCH PAPERS Kim, S.-Y. et al. Diverging neural pathways assemble a behavioural state from separable features in anxiety. Nature 20 Mar 2013 (doi:10.1038/nature12018) | Jennings, J. H. et al. Distinct extended amygdala circuits for divergent motivational states. Nature 20 Mar 2013 (doi:10.1038/nature12041) 